\title{
A Bayesian Weibull survival model for time to infection data measured with delay
}

Kostoulas, Polychronis; Nielsen, Søren Saxmose; Browne, William; Leontides, Leonidas

Publication date:

2009

Document version

Publisher's PDF, also known as Version of record

Citation for published version (APA):

Kostoulas, P., Nielsen, S. S., Browne, W., \& Leontides, L. (2009). A Bayesian Weibull survival model for time to infection data measured with delay. Poster session presented at Annual meeting of the Society for Veterinary Epidemiology and Preventive Medicine, London, United Kingdom. 


\title{
BAYESIAN WEIBULL SURVIVAL ANALYSIS FOR TIME TO INFECTION DATA MEASURED WITH DELAY
}

\author{
Kostoulas P. a, Nielsen S.S. ${ }^{b}$, Browne W.J. c, Leontides La.
}

Laboratory of Epidemiology, Biostatistics and Animal Health Economics, University of Thessaly, Trikalon 224, GR-43100, Karditsa, Greece bepartment of Large Animal Sciences, University of Copenhagen, Grønnegårdsvej 8, DK-1870 Frederiksberg C, Denmark 'School of Clinical Veterinary Sciences, University of Bristol, BS40 5DU, UK.

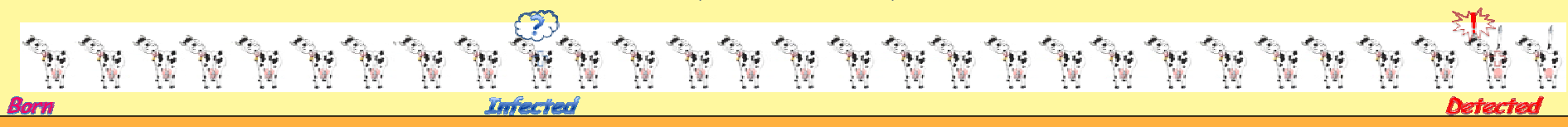

Rational

Unless a perfect diagnostic test exists, time to detection of infection is systematically longer than time to infection. Hence, to infer about the time-toinfection risk, survival analysis models should adjust for the delayed detection of infection. We developed a Bayesian Weibull survival model that adjusts for the delayed detection of infection and demonstrate its use with data from naturally infected with MAP dairy cattle.

\section{Definitions}

Time to infection (tr): The time interval required for the specific component cause to induce infection. The specific component cause comprises of a set of minimal conditions and events (e.g. the single, continuous or repetitive adequate exposure to the pathogen) sufficient to inevitably produce infection.

Time to detection of infection $(t)$ : The time required for detection of the pathogen and/or the immune response of the host.

Detection delay (u): The time interval between (tr) and ( $t$ ).

\section{Bayesian mode}

For the $i^{\text {th }}$ individual, let the unobserved $t r_{i}$ (e.g. time to MAP infection) be equal to:

$$
t r_{i}=t_{i}-u_{i}
$$

We assume that detection delay follows an approximately normal distribution

$$
u_{i} \sim N\left(m_{u_{i}}, \operatorname{tau}_{u_{i}}\right)
$$

with $m_{u,}$ the expected mean of $u$ and $t_{a u_{i}}$ the precision of the normal distribution

For either censored or uncensored observations, we assume that $t r_{i}$ follow a truncated Weibull distribution, with the lower bound (lb) corresponding to zero or the censoring time:

$$
\operatorname{tr}_{i} \sim W\left(\lambda_{i}, \rho\right),[l b,+\infty)
$$

with $\lambda_{i}=e^{b^{*} z_{i}} z_{i}$ the covariate vector for the $i^{\text {th }}$ individual, $b$ a vector of unknown regression coefficients and $\rho$ the shape parameter of the Weibull distribution.

Given the observed distribution of the $t_{i}$ and specifying the prior information on $u_{i}$ the model is fully specified. Adjusted median survival times $\left(m_{i}\right)$ for individuals with specific covariate information $z_{i}$ can be estimated by:

$$
m_{i}=\left(\ln 2 e^{-b z_{i}}\right)^{1 / \rho}
$$

Models were run in the freeware program WinBUGS. For all simulations and applications convergence diagnostics of the MCMC chain revealed no convergence problems

\section{Simulation- Sensitivity analysis}

The impact of ignoring $u$ on $\rho$ and the regression coefficients depended on (a) the longevity and $(b)$ the presence of differenial or non-differential detection delay, (c) the prevalence and (d) the strength of association with the risk factor.

In all considered scenarios model parameters were accurately estimated as long as the true u value was included in the central $90^{\text {th }}$ prior probability space.

\section{Application}

We utilized the proposed model to assess the risk of MAP infection in Danish dairy cattle by analyzing available time to milk seropositivity data. Detailed data information are in Nielsen and Ersboll (2006).

To use our model we needed to specify prior information about the time it takes from MAP infection to get a milk ELISA positive result. Based on available information and expert opinion (N.S.S.), we chose a prior value of $u$ equal to 1300 days ( 3.5 years) extending from 1000 (2.7 years) to 1600 (4.4 years) days: That is a normal $N \sim\left(1300,1 / 4.4 \times 10^{-5}\right)$.

We first ran a standard Weibull model and subsequently our (II) model.

\section{Results}

Low or heavy shedders posed a higher risk to test milk-ELISA positive and were infected earlier in their lives than non-shedders. There was no difference between heavy and low shedders.

Heavy or low MAP shedders are likely to get infected earlier in their lives than non-shedders.

The shape parameter $\rho$ of the weibull distribution was $\rho=2.67>1$ and $\rho=0.56<1$. Thus, the incidence of seroconversion increases (Fig. 1), while the incidence of infection decreases with age (Fig. 2), respectively.

\section{Discussion}

Young calves are more susceptible to MAP infection and resistance to infection increases with aging (Fig. 2), while older animals are more likely to become seropositive (Fig. 1).

Ignoring detection delay can have a severe impact on the estimated risk and median survival time. The proposed model led to corrected estimates and can be particularly useful in the case of chronic infections with a long latent infection

\begin{tabular}{|c|c|c|c|c|c|c|}
\hline 10 & it & i & I & & \multicolumn{2}{|c|}{$\begin{array}{l}\text { - Non-shedders } \\
\text { - Low-shedders } \\
\text { A Heavy-shedders }\end{array}$} \\
\hline & & & I & I & & \\
\hline 0.6 & & & 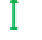 & & I & \\
\hline 0.4 & & & & & & $I$ \\
\hline 0.2 & & & & & I & $\frac{1}{1}$ \\
\hline 0 & 500 & 1000 & $\begin{array}{l}1500 \\
\text { Days }\end{array}$ & 2000 & 2500 & 3000 \\
\hline
\end{tabular}
period.

Figure 1. Predicted median (CrIs) probability of giving a milk-ELISA negative test with time for non-, low- and heavy- MAP shedders.

Figure 2. Predicted median (CrIs) probability of not being infected, with time for non-, low- and heavy-MAP shedders.

Non-shedders

- Low-shedders

A Heavy-shedders

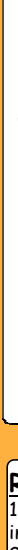

\section{References}

1.Nielsen, S.S., Ersbøll, A.K., 2006. Age at occurrence of Mycobacterium avium subspecies paratuberculosis in naturally infected dairy cows. J Dairy Sci. 89, 4557-66.

2.Spiegelhalter, D.J., Thomas, A., Best, N.G., 2003. WinBUGS Version 1.4 User Manual. MRC Biostatistics Unit. 